\title{
Review: interactive, but not didactic, continuing medical education is effective in changing physician performance
}

\author{
Davis D, Thomson O'Brien MA, Freemantle N, et al. Impact of formal continuing medical education. Do conferences, \\ workshops, rounds, and other traditional continuing education activities change physician behavior or health care \\ outcomes? JAMA 1999 Sep 1;282:867-74.
}
QUESTION: How effective are formal continuing medical education (CME) interventions in changing physician performance and health care outcomes?

\section{Data sources}

Studies were identified by using the Research and Development Resource Base in CME at the University of Toronto, the Specialised Register of the Cochrane Effective Practice and Organisation of Care Group, Medline (1993 to January 1999), CINAHL, ERIC, EMBASE/Excerpta Medica, and PsycINFO and by searching bibliographies of relevant papers.

\section{Study selection}

Studies were selected if they were randomised controlled trials; if they used formal CME interventions of a didactic, an interactive, or a mixed didactic and interactive nature; if they objectively determined either physician performance in the workplace or health care outcomes, or both; and if $\geq 50 \%$ of the participants were practising physicians. Didactic interventions were those that offered minimal participant interaction (eg, lectures or presentations); interactive interventions were those that encouraged participant activity and provided the opportunity to practice skills (eg, role playing, discussion groups, hands on training, or problem or case solving). Studies were excluded if they used coercive educational activities or provided incentives for learning.

\section{Data extraction}

Methodological quality, participant characteristics, nature of intervention (didactic, interactive, or mixed), occurrence of intervention (1 time or series), educational content and format, intervention group size, physician performance, and health care outcomes.

\section{Main results}

64 studies were identified. 14 studies (17 interventions) met the selection criteria. 9 of 17 interventions that assessed physician performance and 3 of 4 interventions that assessed health care outcomes showed positive changes in $\geq 1$ measure. Meta-analysis of 7 studies showed no overall effect of CME interventions (standardised weighted mean difference [WMD] $0.34,95 \%$ CI -0.22 to 0.97 ). A meta-analysis that included only interactive and mixed CME interventions showed an effect on physician performance (standardised WMD 0.67, CI 0.01 to 1.45). None of the 4 didactic CME interventions altered physician performance. No association between intervention group size and positive outcomes was shown.

\section{Conclusion}

Formal interactive, but not didactic, continuing medical education interventions are effective in changing physician performance.

\section{COMMENTARY}

The importance of CME is acknowledged by medical teaching institutions, certifying and licensing bodies, and practising physicians; but the problem is how to deliver an effective product. In Europe, the trend seems to be toward more formalised CME based on traditional formats (ie, having formats that are easy to quantify, such as didactic sessions, and paying less attention to contemporary knowledge about how physicians learn and change).

In Norway, we have recertification for general practitioners (GPs) only. GPs who do not meet CME requirements may see a $20 \%$ drop in their annual income, but their license to practice is not jeopardised. Linking CME and continuous quality improvement has been a pioneering development.

The conclusions drawn by Davis and colleagues in this review are similar to those in their previous publications. ${ }^{2}$ All are high quality reviews that examined difficult and important issues. They provide evidence for a redirection of formal CME toward the practice place as a learning arena, the empowering of the learner, and the value of learning that occurs from day to day patient encounters. Furthermore, the broader concept of continuous quality improvement reminds us that other areas of competence (eg, managerial, social, and personal) also need to be nurtured.

The implications are that CME providers need to re-examine their role and be more supportive of practice based learning and that any monitoring system must be tuned to support and document activities that have been shown to promote learning and change.

Hans A Holm, MD, PhD Norwegian Medical Association Oslo, Norway

1 Holm HA. Ouality issues in continuing medical education. BMJ 1998;316:621-4.

2 Davis DA, Thomson MA, Oxman AD, et al. Evidence for the effectiveness of CME. A review of 50 randomized controlled trials. JAMA 1992;268:1111-17.

3 Davis DA, Thomson MA, Oxman AD, et al. Changing physician performance. A systematic review of the effect of continuing medical education strategies. JAMA 1995;274:700-5. 